\title{
Cinética de secagem e determinação do coeficiente de difusão efetivo das polpas de
}

\section{noni e umbu}

\author{
Drying kinetics and determination of the effective diffusion coefficient of noni and umbu pulps \\ Cinética de secado y determinación del coeficiente de difusión efectiva de pulpas noni y umbu
}

Karoline Thays Andrade Araújo ORCID: https://orcid.org/0000-0002-9356-3288 Universidade Federal de Campina Grande, Brasil E-mail: karoline_thays@hotmail.com

Alexandre José de Melo Queiroz ORCID: https://orcid.org/0000-0002-6880-5951 Universidade Federal de Campina Grande, Brasil E-mail: alexandrejmq@gmail.com

Rossana Maria Feitosa de Figueirêdo ORCID: https://orcid.org/0000-0002-6187-5826 Universidade Federal de Campina Grande, Brasil E-mail: rossanamff@gmail.com

Renato Costa da Silva ORCID: https://orcid.org/0000-0003-3172-4776 Universidade Federal de Campina Grande, Brasil E-mail: renatinocosta@gmail.com

Lumara Tatiely Santos Amadeu ORCID: https://orcid.org/0000-0001-7969-2959 Universidade Federal de Campina Grande, Brasil E-mail: lumaratatielyea@gmail.com

Yaroslávia Ferreira Paiva ORCID: https://orcid.org/0000-0002-2096-2122 Universidade Federal de Campina Grande, Brasil E-mail: yaroslaviapaiva@gmail.com

\begin{abstract}
Resumo
O noni (Morinda citrifolia L.) e o umbu (Spondias tuberosa Arruda Câmara) são frutas ainda pouco conhecidas e com exploração industrial em nível incipiente. Para preservar a produção, quando não consumidas assim que colhidas, é necessário submetê-las a processamentos, entre os quais o resfriamento ou a secagem a fim de mantê-las em condições apropriadas para o consumo. Diante disto, objetivou-se com este trabalho avaliar a cinética de secagem das polpas combinadas de noni com umbu nas temperaturas de $40,50,60$ e $70{ }^{\circ} \mathrm{C}$, ajustar modelos matemáticos para descrever o processo, bem como determinar suas propriedades termodinâmicas. Para cada temperatura foram utilizados $200 \mathrm{~g}$ de polpa combinada, secadas por convecção em estufa com circulação forçada de ar, em triplicata. As razões de umidade foram calculadas e os modelos matemáticos de Page, Henderson e Pabis, Logarítmico e Newton foram ajustados para cada temperatura. $\mathrm{O}$ aumento da temperatura do ar de secagem promoveu redução no tempo necessário para a remoção de água das polpas combinadas de noni com umbu. O modelo de Page apresentou o maior valor de coeficiente de determinação ajustado $(>0,99)$ e o menor valor do desvio quadrático médio $(<0,026)$, em quase todas as temperaturas. A elevação da temperatura do ar de secagem promoveu o aumento do coeficiente de difusão efetivo.
\end{abstract}

Palavras-chave: Morinda citrifolia L.; Spondias tuberosa Arruda Câmara; Difusividade.

\begin{abstract}
Noni (Morinda citrifolia L.) and umbu (Spondias tuberosa Arruda Câmara) are still little-known fruits with industrial exploitation at an incipient level. To preserve production, when not consumed as soon as harvested, it is necessary to subject them to processing, including cooling or drying in order to keep them in conditions suitable for consumption. In view of this, the objective of this work was to evaluate the drying kinetics of the combined pulps of noni with umbu at temperatures of $40,50,60$ and $70^{\circ} \mathrm{C}$, to adjust mathematical models to describe the process, as well as to determine their thermodynamic properties. For each temperature, 200g of combined pulp were used, dried by convection in an oven with forced air circulation, in triplicate. The humidity ratios were calculated and the mathematical models of Page, Henderson and Pabis, Logarithmic and Newton were adjusted for each temperature. The increase in the temperature of the drying air reduced the time needed to remove water from the combined pulps of noni and umbu. The Page model showed the highest value of the adjusted coefficient of determination (>0.99) and
\end{abstract}


the lowest value of the mean square deviation $(<0.026)$, at almost all temperatures. The increase in the temperature of the drying air promoted an increase in the effective diffusion coefficient.

Keywords: Morinda citrifolia L.; Spondias tuberosa Arruda Câmara; Diffusivity.

\section{Resumen}

Noni (Morinda citrifolia L.) y umbu (Spondias tuberosa Arruda Câmara) son frutos aún poco conocidos con explotación industrial a un nivel incipiente. Para conservar la producción, cuando no se consumen tan pronto como se recolectan, es necesario someterlos a procesamiento, incluyendo enfriamiento o secado para mantenerlos en condiciones aptas para el consumo. Ante esto, el objetivo de este trabajo fue evaluar la cinética de secado de las pulpas combinadas de noni con umbu a temperaturas de 40, 50, 60 y $70{ }^{\circ} \mathrm{C}$, para ajustar modelos matemáticos para describir el proceso, así como para determinar sus propiedades termodinámicas. Para cada temperatura se utilizaron 200 g de pulpa combinada, secada por convección en estufa con circulación de aire forzado, por triplicado. Se calcularon las relaciones de humedad y se ajustaron los modelos matemáticos de Page, Henderson y Pabis, Logarithmic y Newton para cada temperatura. El aumento de la temperatura del aire de secado redujo el tiempo necesario para eliminar el agua de las pulpas combinadas de noni y umbu. El modelo de Page mostró el valor más alto del coeficiente de determinación ajustado $(>0,99)$ y el valor más bajo de la desviación cuadrática media $(<0,026)$, a casi todas las temperaturas. El aumento de la temperatura del aire de secado promovió un aumento del coeficiente de difusión efectivo.

Palabras clave: Morinda citrifolia L.; Spondias tuberosa Arruda Câmara; Difusividad.

\section{Introdução}

Noni é o fruto da planta Morinda citrifolia L., destacando-se entre as frutas classificadas como exóticas devido ao seu alto valor nutricional; pertence à família Rubiaceae com origem no Sudeste Asiático. Cresce tanto em florestas tropicais como em terrenos rochosos ou arenosos, adaptando-se, assim, às características edafoclimáticas do Brasil. O fruto é oval, apresenta cor verde, mudando para amarela e, por fim, quase branca, quando é colhido, atinge de 4 a $7 \mathrm{~cm}$ no maior dos eixos, contém muitas sementes e possui forte odor.

Dentre as frutas nativas do Brasil destaca-se o fruto do umbuzeiro (Spondias tuberosa Arruda Câmara), com sabor marcante e acidez elevada, é uma planta nativa da caatinga, totalmente adaptada às condições de clima e solo do semiárido brasileiro. O umbu é arredondado, com cerca de 3 a 3,5cm de diâmetro, de casca lisa ou com pequenos pelos, que lhe conferem uma textura levemente aveludada, com coloração verde-amarelada, sabor ácido e odor agradável, sendo rico em vitamina C.

A qualidade dos produtos agrícolas, é função dos fatores pré-colheita, da colheita propriamente dita e da pós-colheita. Na fase de pós-colheita, a secagem é um processo tradicional na conservação, responsável por diminuir a disponibilidade de água para reações de deterioração, assegurando a qualidade e estabilidade do produto, considerando que a diminuição da quantidade de água do material reduz a atividade biológica e as mudanças químicas e físicas que ocorrem durante o armazenamento (Celma et al., 2011; Botelho et al., 2015; Casarin et al., 2016).

A cinética de secagem fornece informações sobre o comportamento da transferência de calor e massa entre o produto e o agente de secagem, normalmente o ar, e pode ser descrita por modelos matemáticos, que possibilitam a obtenção de estimativas do tempo necessário para redução do teor de água do produto, em diferentes condições de secagem (Guedes et al., 2000; Sousa et al., 2011).

A partir da modelagem das curvas de secagem de um determinado produto é possível determinar índices físicos e termodinâmicos relacionados às transferências de calor e massa, dentre eles o coeficiente de difusão. Este índice possibilita avaliar e comparar a velocidade de secagem de produtos com tamanhos, formas e texturas diferentes. Já os índices termodinâmicos, que expressam a dependência do coeficiente de difusão com a temperatura, possibilitam avaliar energeticamente o processo de secagem (Botelho et al., 2015), além de ser importante fonte de informação para projetar equipamentos de secagem, calcular a energia requerida no processo, estudar as propriedades da água adsorvida e avaliar a microestrutura e os fenômenos físicos que ocorrem na superfície dos produtos (Corrêa et al., 2010). 
Considerando a importância do estudo teórico do processo de secagem dos produtos agrícolas, este trabalho foi desenvolvido com o objetivo de obter as curvas de secagem dos combinados de polpa de noni com umbu utilizando-se de modelos matemáticos, bem como obter o coeficiente de difusão efetivo e as propriedades termodinâmicas durante a desidratação dos combinados.

\section{Metodologia}

\section{Obtenção dos frutos}

O trabalho foi desenvolvido no Laboratório de Armazenamento e Processamento de Produtos Agrícolas (LAPPA) da Unidade Acadêmica de Engenharia Agrícola (UAEA), na Universidade Federal de Campina Grande (UFCG).

Os frutos de noni (Morinda citrifolia L.) e umbu (Spondias tuberosa Arruda Câmara) em estágio de maturação maduros, foram adquiridos em feira livre. O frutos foram lavados em água corrente, sanitizados em solução de hipoclorito de sódio a 100 ppm por 15 minutos e despolpados separadamente em despolpadeira de aço inoxidável. As polpas integrais obtidas foram fracionadas, embaladas em sacos plásticos de polietileno de baixa densidade, lacradas, identificadas e armazenadas em freezer a $-20{ }^{\circ} \mathrm{C}$ até o momento das análises.

\section{Preparação das amostras}

As polpas combinadas (formulações) foram elaboradas a partir da mistura das polpas integrais de noni e umbu em diferentes proporções: $\mathrm{C}_{1}-25 \%$ de polpa de noni com $75 \%$ de polpa de umbu; $\mathrm{C}_{2}-50 \%$ de polpa de noni com 50\% de polpa de umbu; e $\mathrm{C}_{3}-75 \%$ de polpa de noni com $25 \%$ de polpa de umbu. Para cada temperatura foi destinado $200 \mathrm{~g}$ dessas polpas combinadas, sendo, então, espalhadas uniformemente em bandejas de inox, formando uma camada de aproximadamente $10 \mathrm{~mm}$ de espessura, mensurada com paquímetro digital.

\section{Cinética de secagem}

Os ensaios de secagem foram realizados, em triplicata, em estufa com circulação forçada de ar, nas temperaturas de 40, 50,60 e $70{ }^{\circ} \mathrm{C}$. As bandejas contendo o produto foram pesadas em intervalos de tempo regulares até atingir massa constante. Após atingir o equilíbrio, foram levadas para estufa a $105{ }^{\circ} \mathrm{C}$ durante $24 \mathrm{~h}$ para determinação da massa seca, e com os dados de perda de massa das amostras durante as secagens foram calculados os teores de água.

Com os valores do teor de água foram calculadas as razões do teor de água, utilizando-se a Equação (1).

$$
\mathrm{RX}=\frac{\mathrm{X}-\mathrm{x}_{\mathrm{eq}}}{\mathrm{x}_{0}-\mathrm{X}_{\mathrm{eq}}}
$$

Em que: $\mathrm{X}$ - teor de água do produto (b.s.); $\mathrm{X}_{0}$ - teor de água inicial (b.s.); $\mathrm{X}_{\mathrm{eq}}$ - teor de água de equilíbrio (b.s.).

Em seguida, foram construídas as curvas de cinética de secagem (razão do teor de água em função do tempo de secagem) e os modelos matemáticos (Tabela 1) Henderson e Pabis (Equação 2), Logarítmico (Equação 3), Page (Equação 4) e Newton (Equação 5) foram ajustados aos dados experimentais utilizando-se o programa computacional Statistica® 7.0, por meio de análise de regressão não linear, pelo método Quasi-Newton.

A qualidade dos ajustes foi avaliado por meio dos valores dos coeficientes de determinação $\left(R^{2}\right)$ e dos desvios quadráticos médios (DQM), de acordo com a Equação (6). 
Tabela 1. Modelos matemáticos ajustados às curvas de cinética de secagem das polpas combinadas de noni com umbu.

\begin{tabular}{|c|c|c|}
\hline $\begin{array}{c}\text { Designação do modelo } \\
\text { matemático }\end{array}$ & Equação & Equação no \\
\hline Henderson e Pabis & $\mathrm{RX}=\mathrm{a} \cdot \exp (-\mathrm{k} \cdot \mathrm{t})$ & 2 \\
\hline Logarítmico & $R X=a \cdot \exp (-k \cdot t)+c$ & 3 \\
\hline Page & $\mathrm{RX}=\exp \left(-\mathrm{k} \cdot \mathrm{t}^{\mathrm{n}}\right)$ & 4 \\
\hline Newton & $\mathrm{RX}=\exp (-\mathrm{k} . \mathrm{t})$ & 5 \\
\hline
\end{tabular}

Em que: RX - razão do teor de água (adimensional); a, c, k, n - constantes dos modelos; $\mathrm{t}$ - tempo (min). Fonte: Autores.

$$
\mathrm{DQM}=\sqrt{\frac{(\text { RXpred-RXexp })^{2}}{\mathrm{n}}}
$$

Em que: DQM - desvio quadrático médio; RXpred - razão do teor de água predito pelo modelo; RXexp - razão do teor de água experimental; $\mathrm{n}$ - número de observações.

\section{Solução analítica da equação de difusão}

A difusividade efetiva ou coeficiente de difusão $\left(\mathrm{D}_{\mathrm{EF}}\right)$ foi determinada usando a equação de difusão de Fick para uma placa plana, descrita pela Equação 7.

$$
\mathrm{RX}=\left(\frac{8}{\pi^{2}}\right) \sum_{\mathrm{n}=0}^{\infty} \frac{1}{(2 \mathrm{n}+1)^{2}} \exp \left(-\frac{(2 \mathrm{n}+1)^{2}}{4} \pi^{2} \frac{\mathrm{D}_{\mathrm{EF}} \mathrm{t}}{\mathrm{L}_{0}{ }^{2}}\right)
$$

Em que: RX - razão do teor de água, adimensional; $\mathrm{D}_{\mathrm{EF}}$ - coeficiente de difusão efetiva, $\mathrm{m}^{2} \mathrm{~s}^{-1} ; \mathrm{L}_{0}$ - espessura do produto, $\mathrm{m} ; \mathrm{n}$ - número de termos da equação; $\mathrm{t}$ - tempo, s.

O número de termos da equação utilizado foi de quatro $(n=4)$, uma vez que para maiores valores de $n$ o coeficiente de difusão efetiva não variou.

A relação do aumento do coeficiente de difusão efetivo com a elevação da temperatura do ar de secagem foi descrita por meio da equação de Arrhenius (Equação 8).

$$
\mathrm{D}_{\mathrm{EF}}=\mathrm{D}_{0} \exp \left(\frac{-\mathrm{E}_{\mathrm{a}}}{\mathrm{RT}_{\mathrm{a}}}\right)
$$

Em que: $\mathrm{D}_{0}$ - fator pré-exponencial, $\mathrm{m}^{2} \mathrm{~s}^{-1} ; \mathrm{E}_{\mathrm{a}}$ - energia de ativação, $\mathrm{kJ} \mathrm{mol}^{-1} ; \mathrm{R}$ - constante universal dos gases, 8,314 J $\mathrm{mol}^{-1} \mathrm{~K}^{-1} ; \mathrm{T}_{\mathrm{a}}$ - temperatura absoluta, $\mathrm{K}$.

A obtenção do valor da energia de ativação possibilitou determinar diferentes parâmetros termodinâmicos para a secagem da polpa de noni com umbu, pelo método descrito por Corrêa et al. (2010), como a entalpia $(\Delta \mathrm{H})$, entropia $(\Delta \mathrm{S})$ e a energia livre de Gibbs $(\Delta \mathrm{G})$, de acordo com as Equações 9, 10 e 11.

$$
\Delta \mathrm{H}=\mathrm{E}_{\mathrm{a}}-\mathrm{RT}
$$




$$
\begin{aligned}
& \Delta \mathrm{S}=\mathrm{R}\left(\ln \mathrm{D}_{0}-\ln \frac{\mathrm{k}_{\mathrm{B}}}{\mathrm{h}_{\mathrm{p}}}-\ln \mathrm{T}\right) \\
& \Delta \mathrm{G}=\Delta \mathrm{H}-\mathrm{T}_{\mathrm{a}} \Delta \mathrm{S}
\end{aligned}
$$

Em que: $\Delta \mathrm{H}$ - entalpia, $\mathrm{J} \mathrm{mol}^{-1} ; \Delta \mathrm{S}$ - entropia, $\mathrm{J} \mathrm{mol}^{-1} ; \Delta \mathrm{G}$ - energia livre de Gibbs, $\mathrm{J} \mathrm{mol}^{-1} ; \mathrm{k}_{\mathrm{B}}$ - constante de Boltzmann, $1,38 \times 10^{-23} \mathrm{~J} \mathrm{~K}^{-1} ; \mathrm{h}_{\mathrm{p}}$ - constante de Planck, $6,626 \times 10^{-34} \mathrm{~J} \mathrm{~s}^{-1}$.

\section{Resultados e Discussão}

São apresentados na Tabela 2 os parâmetros dos modelos matemáticos Page, Logarítmico, Henderson e Pabis e Newton, ajustados aos dados experimentais da cinética de secagem das polpas combinadas e seus respectivos coeficientes de determinação $\left(\mathrm{R}^{2}\right)$ e desvios quadráticos médios (DQM).

Tabela 2. Parâmetros de ajuste dos modelos Page, Logarítmico, Henderson e Pabis e Newton, com seus respectivos coeficientes

\begin{tabular}{|c|c|c|c|c|c|c|}
\hline \multicolumn{7}{|c|}{ Page } \\
\hline \multirow{2}{*}{$\begin{array}{c}\text { Temperatura } \\
\left({ }^{\circ} \mathbf{C}\right)\end{array}$} & \multirow{2}{*}{ Combinados } & \multicolumn{3}{|c|}{ Parâmetros } & \multirow{2}{*}{$\mathbf{R}^{2}$} & \multirow{2}{*}{ DQM } \\
\hline & & $\mathbf{k}$ & & $\mathbf{n}$ & & \\
\hline \multirow{3}{*}{40} & $\mathrm{C}_{1}$ & 0,0009 & & 1,3441 & 0,9982 & 0,0161 \\
\hline & $\mathrm{C}_{2}$ & 0,0006 & & 1,3784 & 0,9980 & 0,0174 \\
\hline & $\mathrm{C}_{3}$ & 0,0003 & & 1,3222 & 0,9815 & 0,0489 \\
\hline \multirow{3}{*}{50} & $\mathrm{C}_{1}$ & 0,0004 & & 1,4777 & 0,9946 & 0,0268 \\
\hline & $\mathrm{C}_{2}$ & 0,0007 & & 1,4033 & 0,9960 & 0,0247 \\
\hline & $\mathrm{C}_{3}$ & 0,0007 & & 1,4428 & 0,9968 & 0,0228 \\
\hline \multirow{3}{*}{60} & $\mathrm{C}_{1}$ & 0,0006 & & 1,5277 & 0,9983 & 0,0157 \\
\hline & $\mathrm{C}_{2}$ & 0,0008 & & 1,4722 & 0,9964 & 0,0237 \\
\hline & $\mathrm{C}_{3}$ & 0,0008 & & 1,4697 & 0,9962 & 0,0246 \\
\hline \multirow{3}{*}{70} & $\mathrm{C}_{1}$ & 0,0013 & & 1,4697 & 0,9959 & 0,0247 \\
\hline & $\mathrm{C}_{2}$ & 0,0018 & & 1,4837 & 0,9964 & 0,0236 \\
\hline & $\mathrm{C}_{3}$ & 0,0010 & & 1,5024 & 0,9968 & 0,0224 \\
\hline \multicolumn{7}{|c|}{ Logarítmico } \\
\hline \multirow{2}{*}{$\begin{array}{c}\text { Temperatura } \\
\left({ }^{\circ} \mathbf{C}\right)\end{array}$} & \multirow{2}{*}{ Combinados } & \multicolumn{3}{|c|}{ Parâmetros } & \multirow{2}{*}{$\mathbf{R}^{2}$} & \multirow{2}{*}{ DQM } \\
\hline & & $\mathbf{a}$ & $\mathbf{k}$ & $\mathbf{c}$ & & \\
\hline \multirow{3}{*}{40} & $\mathrm{C}_{1}$ & 1,1572 & 0,0044 & 0,1171 & 0,9940 & 0,0270 \\
\hline & $\mathrm{C}_{2}$ & 1,1849 & 0,0037 & 0,1428 & 0,9929 & 0,0300 \\
\hline & $\mathrm{C}_{3}$ & 1,9804 & 0,0008 & $-0,9773$ & 0,9941 & 0,0276 \\
\hline \multirow{3}{*}{50} & $\mathrm{C}_{1}$ & 1,4425 & 0,0028 & 0,4079 & 0,9918 & 0,0328 \\
\hline & $\mathrm{C}_{2}$ & 1,1456 & 0,0051 & 0,1019 & 0,9885 & 0,0418 \\
\hline & $\mathrm{C}_{3}$ & 1,1132 & 0,0061 & $-0,0568$ & 0,9862 & 0,0472 \\
\hline \multirow{3}{*}{60} & $\mathrm{C}_{1}$ & 1,1946 & 0,0064 & 0,1294 & 0,9866 & 0,0443 \\
\hline & $\mathrm{C}_{2}$ & 1,1213 & 0,0076 & 0,0621 & 0,9852 & 0,0477 \\
\hline & $\mathrm{C}_{3}$ & 1,1078 & 0,0077 & $-0,0447$ & 0,9844 & 0,0498 \\
\hline \multirow{3}{*}{70} & $\mathrm{C}_{1}$ & 1,1499 & 0,0101 & 0,0771 & 0,9950 & 0,0471 \\
\hline & $\mathrm{C}_{2}$ & 1,1412 & 0,0077 & 0,0761 & 0,9842 & 0,0492 \\
\hline & $\mathrm{C}_{3}$ & 1,1174 & 0,0101 & $-0,0355$ & 0,9848 & 0,0491 \\
\hline \multicolumn{7}{|c|}{ Henderson e Pabis } \\
\hline \multirow{2}{*}{$\begin{array}{c}\text { Temperatura } \\
\left({ }^{\circ} \mathbf{C}\right)\end{array}$} & \multirow{2}{*}{ Combinados } & \multicolumn{3}{|c|}{ Parâmetros } & \multirow{2}{*}{$\mathbf{R}^{2}$} & \multirow{2}{*}{ DQM } \\
\hline & & $\mathbf{a}$ & & $\mathbf{k}$ & & \\
\hline 40 & $\mathrm{C}_{1}$ & 1,0593 & & 0,0056 & 0,9868 & 0,0422 \\
\hline
\end{tabular}
de determinação $\left(\mathrm{R}^{2}\right)$ e desvios quadráticos médios $(\mathrm{DQM})$ da cinética de secagem dos combinados de noni e umbu. 


\begin{tabular}{|c|c|c|c|c|c|}
\hline & $\mathrm{C}_{2}$ & 1,0635 & 0,0049 & 0,9838 & 0,0486 \\
\hline & $\mathrm{C}_{3}$ & 1,0474 & 0,0024 & 0,9632 & 0,0690 \\
\hline \multirow{3}{*}{50} & $\mathrm{C}_{1}$ & 1,0698 & 0,0051 & 0,9690 & 0,0639 \\
\hline & $\mathrm{C}_{2}$ & 1,0607 & 0,0063 & 0,9810 & 0,0536 \\
\hline & $\mathrm{C}_{3}$ & 1,0669 & 0,0068 & 0,9822 & 0,0536 \\
\hline \multirow{3}{*}{60} & $\overline{C_{1}}$ & 1,0844 & 0,0081 & 0,9773 & 0,0576 \\
\hline & $\mathrm{C}_{2}$ & 1,0702 & 0,0086 & 0,9808 & 0,0543 \\
\hline & $\mathrm{C}_{3}$ & 1,0711 & 0,0084 & 0,9814 & 0,0544 \\
\hline \multirow{3}{*}{70} & $\mathrm{C}_{1}$ & 1,0863 & 0,0118 & 0,9795 & 0,0551 \\
\hline & $\mathrm{C}_{2}$ & 1,0780 & 0,0089 & 0,9786 & 0,0571 \\
\hline & $\mathrm{C}_{3}$ & 1,0882 & 0,0109 & 0,9826 & 0,0525 \\
\hline \multicolumn{6}{|c|}{ Newton } \\
\hline \multirow{2}{*}{$\begin{array}{c}\text { Temperatura } \\
\left({ }^{\circ} \mathbf{C}\right)\end{array}$} & \multirow{2}{*}{ Combinados } & \multicolumn{2}{|c|}{ Parâmetros } & $\mathbf{P}^{2}$ & $\mathrm{DOM}$ \\
\hline & & \multicolumn{2}{|c|}{$\mathbf{k}$} & $\mathbf{k}$ & DQIn \\
\hline \multirow{3}{*}{40} & $\mathrm{C}_{1}$ & \multicolumn{2}{|c|}{0,0051} & 0,9805 & 0,0513 \\
\hline & $\mathrm{C}_{2}$ & \multicolumn{2}{|c|}{0,0045} & 0,9764 & 0,0586 \\
\hline & $\mathrm{C}_{3}$ & \multicolumn{2}{|c|}{0,0021} & 0,9576 & 0,0742 \\
\hline \multirow{3}{*}{50} & $\overline{C_{1}}$ & \multicolumn{2}{|c|}{0,0046} & 0,9579 & 0,0745 \\
\hline & $\mathrm{C}_{2}$ & \multicolumn{2}{|c|}{0,0058} & 0,9751 & 0,0615 \\
\hline & $\mathrm{C}_{3}$ & \multicolumn{2}{|c|}{0,0062} & 0,9761 & 0,0620 \\
\hline \multirow{3}{*}{60} & $\mathrm{C}_{1}$ & \multicolumn{2}{|c|}{0,0072} & 0,9653 & 0,0713 \\
\hline & $\mathrm{C}_{2}$ & \multicolumn{2}{|c|}{0,0079} & 0,9740 & 0,0633 \\
\hline & $\mathrm{C}_{3}$ & \multicolumn{2}{|c|}{0,0077} & 0,9750 & 0,0630 \\
\hline \multirow{3}{*}{70} & $\mathrm{C}_{1}$ & \multicolumn{2}{|c|}{0,0106} & 0,9689 & 0,0678 \\
\hline & $\mathrm{C}_{2}$ & \multicolumn{2}{|c|}{0,0081} & 0,9698 & 0,0679 \\
\hline & $\mathrm{C}_{3}$ & \multicolumn{2}{|c|}{0,0097} & 0,9737 & 0,0645 \\
\hline
\end{tabular}

Fonte: Autores.

Os modelos matemáticos avaliados se ajustaram bem aos dados experimentais de secagem para a faixa de temperatura estudada, com $\mathrm{R}^{2}$ superiores a 0,95 e DQM inferiores a 0,07 podendo ser utilizados na predição das cinéticas de secagem das polpas combinadas. Entretanto, dentre os modelos avaliados, o que melhor se ajustou aos dados experimentais foi o de Page, apresentando os maiores coeficientes de determinação $\left(R^{2}\right)$, variando entre 0,9815 e 0,9983 , os menores desvios quadráticos médios (DQM), variando entre 0,0157 e 0,0489 para a faixa de temperatura de 40 a $70{ }^{\circ} \mathrm{C}$, o que indica que este modelo representa satisfatoriamente o fenômeno da secagem das formulações de noni e umbu.

Valores semelhantes de $\mathrm{R}^{2}$ e de DQM para o modelo de Page foram observados por Sousa et al. (2015) ao realizarem a secagem de Spondias sp. em camada fina, nas temperaturas de 50, 60 e $70{ }^{\circ} \mathrm{C}$; e Silva et al. (2015), ao realizarem o estudo experimental da secagem de polpa de Achachairu em camada fina nas temperaturas de 50,60 e $70{ }^{\circ} \mathrm{C}$; e ainda Gonçalves et al. (2016) ao secarem a polpa de banana verde (Musa acuminata) em estufa com temperaturas de 55, 65 e $75{ }^{\circ} \mathrm{C}$. Pê et al. (2016), ao realizarem a modelagem matemática da secagem em camada de espuma de polpa de caqui, obtiveram $\mathrm{R}^{2}>0,96$ e desvios quadráticos médios inferiores a 0,01, para os modelos de Page, Logarítmico e Midilli.

Constata-se, de maneira geral, que o parâmetro "k" (constante de secagem) dos modelos Page, Logarítmico, Henderson e Pabis e Newton apresentaram tendência de aumento com o aumento da temperatura de secagem. Esta constante está relacionada à difusividade térmica no processo de secagem e seu comportamento ganha sentido, pois o aumento da temperatura fornece maior quantidade de energia na forma de calor, o que faz com que a amostra se ajuste mais rapidamente à temperatura em seu entorno, atingindo o teor de água de equilíbrio em menor tempo do que no uso de temperaturas mais baixas (Gupta et al., 2011). Silva Filho et al. (2016) ao realizarem a modelagem matemática da cinética de secagem da polpa de manga cv. Espada também 
observaram que os parâmetros "k" para os modelos de Henderson e Pabis, Logarítmico e Page aumentaram com o aumento da temperatura de secagem.

O parâmetro "n" do modelo de Page, exibindo valores de 1,3441 a 1,5277, que segundo Araújo et al. (2017), reflete a resistência interna do produto à secagem, aumentou com o aumento da temperatura de secagem. Comportamento também verificado por Sousa et al. (2011) ao realizarem a modelagem matemática para descrição da cinética de secagem de polpa de oiti (Licania tomentosa) nas temperaturas de 50,60 e $70{ }^{\circ} \mathrm{C}$.

Verifica-se que o parâmetro "a" do modelo de Henderson e Pabis aumentou com o aumento da temperatura de secagem. Comportamento também verificado por Castro et al. (2016) ao ajustarem o modelo de Henderson e Pabis aos dados experimentais da cinética de secagem de polpa de taturubá (Pouteria macrophylla (lam.) eyma.) em camada fina nas temperaturas de 50 a 70 ${ }^{\circ} \mathrm{C}$. Entretanto, para o modelo Logarítmico o parâmetro "a" não teve um comportamento de aumento ou redução com aumento da temperatura.

A Figura 1 ilustra as curvas ajustadas com o modelo matemático de Page aos dados experimentais da cinética de secagem das polpas combinadas nas temperaturas de $40,50,60$ e $70{ }^{\circ} \mathrm{C}$. Quando as polpas combinadas de noni e umbu foram secadas a $40{ }^{\circ} \mathrm{C}$, o tempo necessário para reduzir o teor de água foi de 640,720 e $940 \mathrm{~min}$; já para $70{ }^{\circ} \mathrm{C}$ o tempo foi de 400,520 e 640 min para os combinados $C_{1}, C_{2}$ e $C_{3}$, respectivamente. À medida que a temperatura aumenta o tempo de secagem diminui e as curvas adquirem uma inclinação maior, visto que que há maior quantidade de calor sendo transferido do ar para o material.

Figura 1. Curvas de cinética de secagem das polpas combinadas $C_{1}$ (a), $C_{2}(b), C_{3}$ (c) nas temperaturas de $40,50,60$ e $70{ }^{\circ}$, com ajustes pelo modelo de Page.

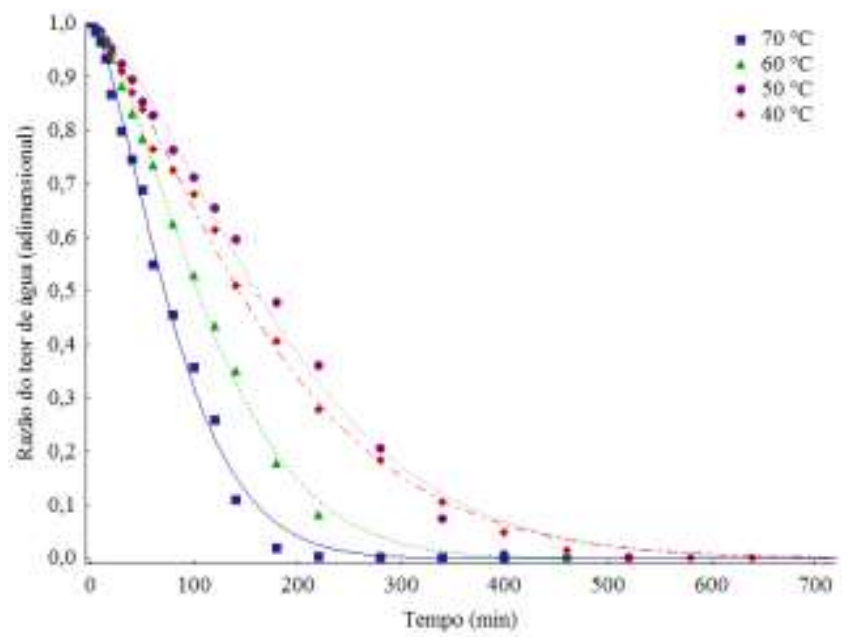

(a)

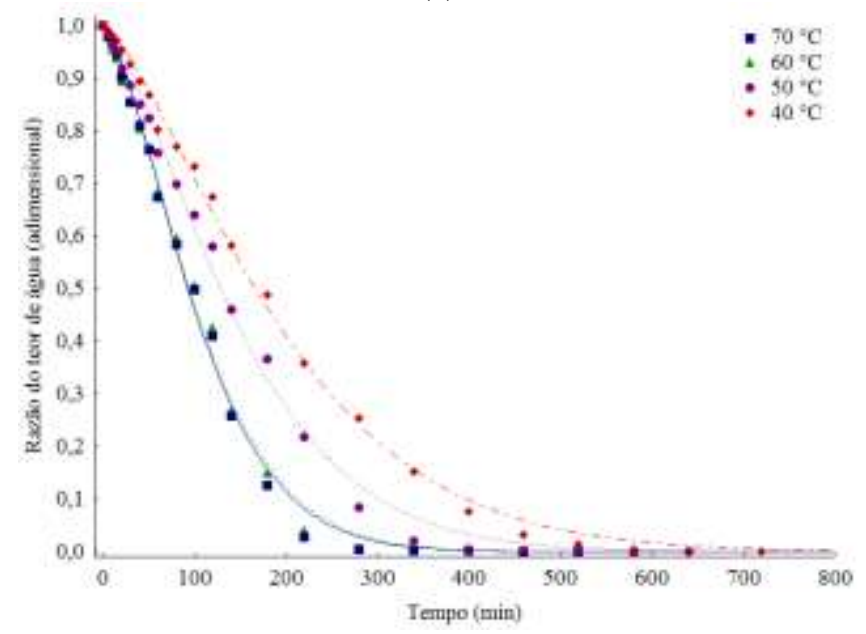


(b)

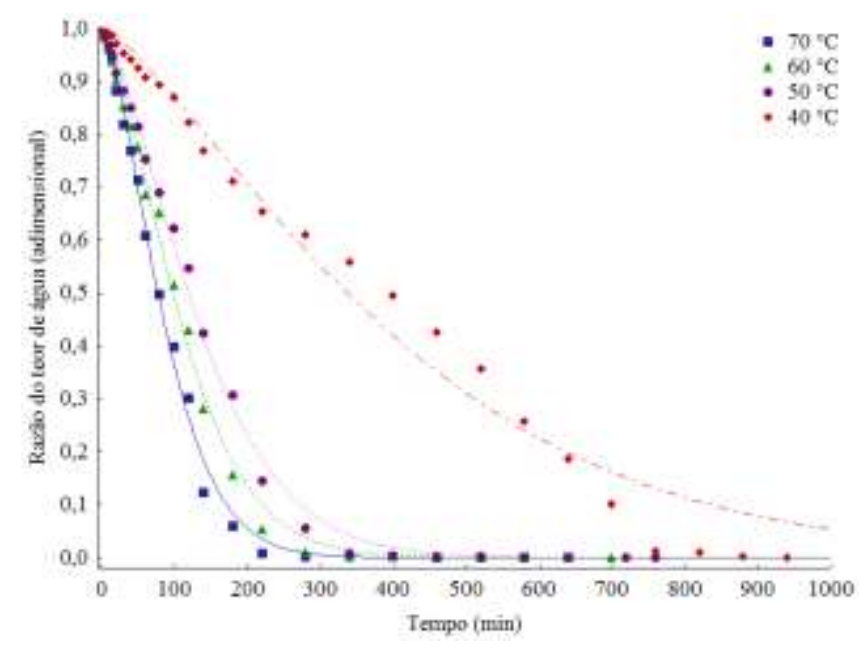

(c)

Fonte: Autores.

O modelo de Page foi considerado o melhor para estimar as curvas de cinética de secagem das polpas combinadas $\mathrm{C}_{1}$, $\mathrm{C}_{2}$, e $\mathrm{C}_{3}$, e sua representação na Figura 1 demonstra a dispersão dos dados estimados em relação aos dados experimentais. Observa-se que as curvas ajustadas ficaram próximas aos dados experimentais, evidenciando os altos valores de $\mathrm{R}^{2}$ e baixos DQM, e que os maiores tempos de secagem foram nas menores temperaturas e as curvas a 60 e $70{ }^{\circ} \mathrm{C}$ ficaram muito próximas. As curvas de cinética de secagem de um produto depende de vários fatores como velocidade do ar de secagem, teor de água inicial, teor de água final, umidade relativa do ar, temperatura, composição, pressão, entre outros (Moreira et al., 2018).

Silva et al. (2016) observaram o efeito da temperatura $\left(40,50\right.$ e $\left.60^{\circ} \mathrm{C}\right)$ sobre as curvas de secagem em camada delgada da polpa de carambola, notando que com o aumento da temperatura do ar de secagem, ocorreu uma elevação das taxas de remoção de água do produto. Dieb et al. (2015) ao determinarem as curvas de cinética de secagem da polpa de graviola pelo processo de secagem em camada de espuma nas temperaturas de 50,60 e $70^{\circ} \mathrm{C}$, confirmaram o efeito da temperatura no sentido de diminuir o tempo de secagem, considerando, todavia, que temperaturas muito elevadas podem comprometer a qualidade do produto. Ferreira et al. (2012) explicaram que a influência da temperatura se dá pelo aumento do potencial de transferência de calor entre o ar e o produto. Silva et al. (2014), ao desidratarem banana em camada fina nas temperaturas de $40,50,60$ e $70{ }^{\circ} \mathrm{C}$, também observaram redução nos tempos de secagem, sendo necessários 3625 minutos para a menor temperatura de secagem e 1200 minutos para a maior temperatura, até as amostras atingirem o teor de água de equilíbrio. Considerando que trata-se do mesmo intervalo de temperatura avaliado neste estudo, evidencia-se a influência do tipo de produto.

Verifica-se, pela Tabela 3, que os valores do coeficiente de difusão efetiva $\left(\mathrm{D}_{\mathrm{EF}}\right)$ aumentaram em resposta a elevação da temperatura do ar de secagem, apresentando valores entre $2,98 \times 10^{-9} \mathrm{e} 8,91 \times 10^{-9} \mathrm{~m}^{2} \mathrm{~s}^{-1}$; este fato é esperado, uma vez que com o incremento da temperatura a viscosidade da água diminui, esta propriedade influi diretamente na resistência do fluido ao escoamento e, portanto, sua diminuição acarreta em maior facilidade de difusão das moléculas de água nos capilares do produto, uma vez que a difusividade indica a velocidade com que a água migra do interior para a superfície do material, sendo então vaporizada (Baptestini et al., 2015; Goneli et al., 2014). Os valores da energia de ativação para a difusão de água nos combinados de polpa de noni e umbu durante o processo de secagem e para a faixa de temperatura estudada estão apresentados ainda na Tabela 3, valores que estão coerentes com os resultados relatados na literatura para secagem de produtos agrícolas, que, de acordo com Zogzas et al. (1996), pode variar de 12,7 a $110 \mathrm{~kJ} \mathrm{~mol}^{-1}$. 
Tabela 3. Valores médios do coeficiente de difusão efetiva ( $\left.\mathrm{D}_{\mathrm{EF}}\right)$ e da energia de ativação (Ea) obtidos na secagem da polpa de noni e umbu nas diferentes combinações e diferentes temperaturas de secagem.

\begin{tabular}{cccc}
\hline Temperatura $\left({ }^{\circ} \mathbf{C}\right)$ & Combinados & DEF $\left(\mathbf{x ~ 1 0} \mathbf{~ m}^{\mathbf{9}} \mathbf{~ s}^{\mathbf{1}}\right)$ & Ea $\left(\mathbf{k J ~} \mathbf{~ m o l}^{\mathbf{1}}\right)$ \\
\hline \multirow{2}{*}{40} & $\mathrm{C}_{1}$ & 4,27 & 21,64 \\
& $\mathrm{C}_{2}$ & 4,09 & 12,68 \\
& $\mathrm{C}_{3}$ & 2,98 & 20,19 \\
\hline \multirow{2}{*}{50} & $\mathrm{C}_{1}$ & 6,29 & 21,30 \\
& $\mathrm{C}_{2}$ & 4,82 & 12,65 \\
& $\mathrm{C}_{3}$ & 4,18 & 19,94 \\
\hline \multirow{2}{*}{60} & $\mathrm{C}_{1}$ & 7,61 & 21,43 \\
& $\mathrm{C}_{2}$ & 5,47 & 12,69 \\
& $\mathrm{C}_{3}$ & 5,13 & 19,98 \\
\hline \multirow{2}{*}{70} & $\mathrm{C}_{1}$ & 8,91 & 21,62 \\
& $\mathrm{C}_{2}$ & 6,27 & 12,68 \\
& $\mathrm{C}_{3}$ & 5,88 & 20,19 \\
\hline
\end{tabular}

Fonte: Autores.

Na Figura 2 apresenta-se a variação do coeficiente de difusão efetivo devido à variação da temperatura do ar de secagem para os combinados $\mathrm{C}_{1}$ (a), $\mathrm{C}_{2}$ (b) e $\mathrm{C}_{3}$ (c), podendo-se constatar que a linearidade decrescente evidencia a uniformidade de variação da taxa de secagem dentro da faixa de temperatura avaliada, além de apresentar elevada correspondência entre os valores observados e os estimados pela relação de Arrhenius. Verifica-se que os valores de Ln(Def), em função do inverso da temperatura absoluta $\left(1 / \mathrm{T}\left(\mathrm{K}^{-1}\right)\right)$, reduziram de $-18,52$ a $70{ }^{\circ} \mathrm{C}$ para $-19,30$ a $40{ }^{\circ} \mathrm{C}$ para o combinado $\mathrm{C}_{1}$. De $-18,89$ a $70{ }^{\circ} \mathrm{C}$ para $-19,34$ a $40{ }^{\circ} \mathrm{C}$ para o combinado $\mathrm{C}_{2}$ e de $-18,95$ a $70{ }^{\circ} \mathrm{C}$ para $-19,64$ a $40{ }^{\circ} \mathrm{C}$ para o combinado $\mathrm{C}_{3}$. Este comportamento também foi observado por Alves et al. (2016) ao secarem a polpa de abacate cv 'Quintal' em camada de espuma nas temperaturas de 50, 60, 70 e $80{ }^{\circ} \mathrm{C}$, pressupondo que a difusividade efetiva, em função da temperatura, controla o processo de secagem em período decrescente. 
Research, Society and Development, v. 10, n. 4, e46710413985, 2021

(CC BY 4.0) | ISSN 2525-3409 | DOI: http://dx.doi.org/10.33448/rsd-v10i4.13985

Figura 2. Representação de Arrhenius para os combinados de polpa de noni e umbu na faixa de temperatura de 40 a $70{ }^{\circ} \mathrm{C}$, equação de regressão e o respectivo coeficiente de determinação.

$$
1 / \mathrm{T}\left(\mathrm{K}^{-1}\right)
$$

$\begin{array}{lllllllllll}0,0029 & 0,0030 & 0,0030 & 0,0031 & 0,0031 & 0,0032 & 0,0032 & 0,0033\end{array}$

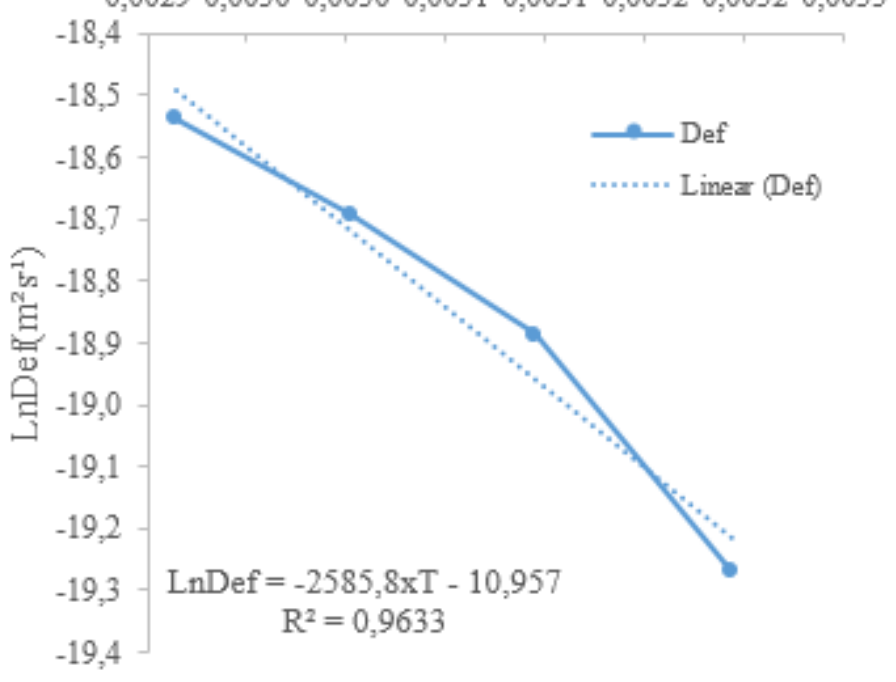

(a) $\mathrm{C}_{1}$

1/T $\left(\mathrm{K}^{-1}\right)$

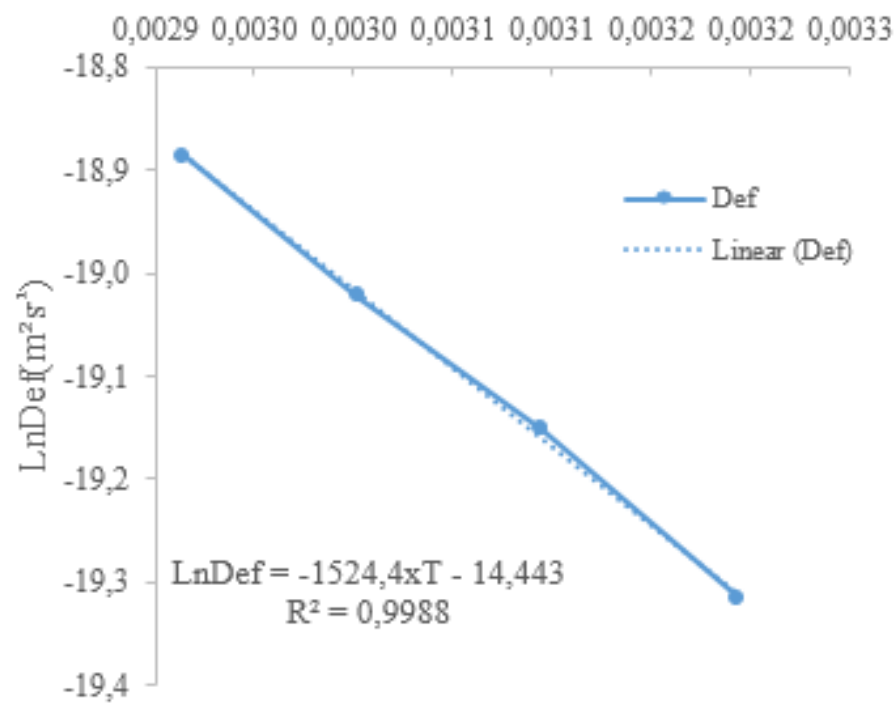

(b) $\mathrm{C}_{2}$ 


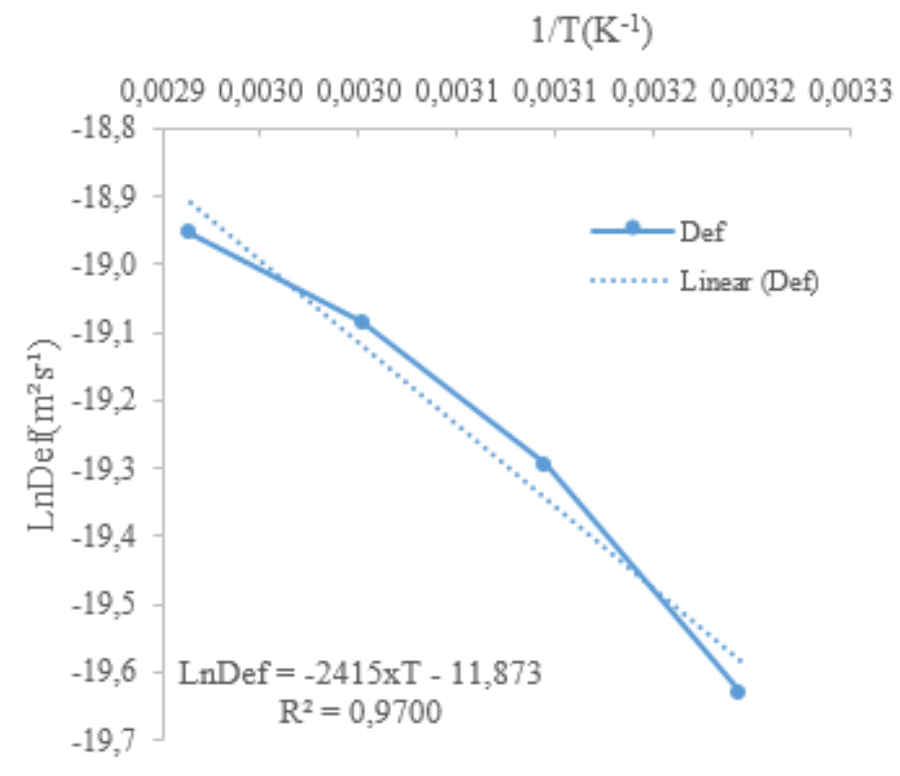

(c) $\mathrm{C}_{3}$

Fonte: Autores.

Na Tabela 4 pode-se observar os valores das propriedades termodinâmicas (entalpia de ativação, entropia de ativação e energia livre de Gibbs) encontradas no processo de secagem dos combinados de polpa de noni e umbu em diferentes temperaturas $\left(40,50,60\right.$ e $\left.70{ }^{\circ} \mathrm{C}\right)$.

Os valores de entalpia sofreram redução durante a secagem dos combinados de polpa de noni e umbu, com o aumento da temperatura do ar. Araújo et al. (2017) trabalhando com a secagem de amendoim em camada delgada também observaram resultado similar. Alves et al. (2016) secaram em camada de espuma a polpa de abacate cv 'Quintal' e verificaram que a entalpia $(\Delta \mathrm{H})$ reduziu de 36,786 para $36,537 \mathrm{~kJ} \mathrm{~mol}^{-1}$ com o aumento da temperatura $\left(50,60,70\right.$ e $\left.80^{\circ} \mathrm{C}\right)$. Segundo Corrêa et al. (2010), isso indica a necessidade de menos energia para remover a água ligada ao produto durante a secagem.

Do comportamento da entropia na Tabela 4, observa-se que, para os diferentes combinados de polpa de noni e umbu, esta propriedade termodinâmica comportou-se de forma similar à entalpia, onde seus valores foram reduzindo com o aumento da temperatura. Moreira et al. (2008) esclarecem que os valores negativos de entropia foram atribuídos à existência de adsorção química e/ou modificações estruturais do adsorvente. Os valores encontrados foram entre -223,11 e -226,17 J mol-1 , Oliveira et al. (2015) relatam que a pequena variação deveu-se à metodologia utilizada, que se baseia nas constantes de Planck e de Boltzmann, sendo a variação de entropia apenas retratada pela variação de temperatura, com os demais parâmetros constantes.

Os valores da energia livre de Gibbs $(\Delta \mathrm{G})$ aumentaram proporcionalmente com o aumento da temperatura do ar de secagem, e seus valores foram positivos, indicando que a secagem nas condições do presente trabalho não foi espontânea. De acordo com Ojediran e Raji (2010), a energia de ativação é uma medida da sensibilidade à temperatura, sendo a energia necessária para iniciar a difusão do líquido no interior do produto agrícola. 
Tabela 4. Valores da entalpia $(\Delta \mathrm{H})$, entropia de ativação $(\Delta \mathrm{S})$ e energia livre de Gibbs de ativação $(\Delta \mathrm{G})$ para a secagem da polpa de noni e umbu nas diferentes combinações e diferentes temperaturas de secagem.

\begin{tabular}{ccccc}
\hline Temperatura $\left({ }^{\circ} \mathbf{C}\right)$ & Combinados & $\Delta{\mathrm{H}\left(\mathrm{J} \mathrm{mol}^{-1}\right)}^{-1}$ & $\Delta \mathrm{S}\left(\mathrm{J} \mathrm{mol}^{-1}\right)$ & $\Delta \mathrm{G}\left(\mathrm{kJ} \mathrm{mol}^{-1}\right)$ \\
\hline \multirow{2}{*}{40} & $\mathrm{C}_{1}$ & 19040,36 & $-225,41$ & 89,628 \\
& $\mathrm{C}_{2}$ & 10080,92 & $-223,11$ & 79,949 \\
& $\mathrm{C}_{3}$ & 17591,11 & $-224,74$ & 87,970 \\
\hline \multirow{2}{*}{50} & $\mathrm{C}_{1}$ & 18612,13 & $-225,67$ & 91,538 \\
& $\mathrm{C}_{2}$ & 9961,35 & $-223,38$ & 82,145 \\
& $\mathrm{C}_{3}$ & 17250,35 & $-225,00$ & 89,961 \\
\hline \multirow{2}{*}{60} & $\mathrm{C}_{1}$ & 18658,30 & $-225,93$ & 93,925 \\
& $\mathrm{C}_{2}$ & 9917,11 & $-223,63$ & 84,419 \\
& $\mathrm{C}_{3}$ & 17213,64 & $-225,26$ & 92,258 \\
\hline \multirow{2}{*}{70} & $\mathrm{C}_{1}$ & 18768,62 & $-226,17$ & 96,379 \\
& $\mathrm{C}_{2}$ & 9826,09 & $-223,88$ & 86,649 \\
& $\mathrm{C}_{3}$ & 17341,46 & $-225,50$ & 94,723 \\
\hline
\end{tabular}

Fonte: Autores.

\section{Conclusão}

O modelo de Page é o que apresenta o melhor ajuste aos dados experimentais da cinética de secagem nas temperaturas de 40, 50, 60 e $70^{\circ} \mathrm{C}$ para as três amostras das polpas combinadas de noni com umbu, sendo o modelo recomendado para descrever as curvas de secagem, nas condições em que foi realizado o experimento.

Para ambas combinações das polpas de noni com umbu, o coeficiente de difusão efetivo ( $\mathrm{D}_{\mathrm{EF}}$ ) aumenta com a elevação da temperatura do ar de secagem, os valores obtidos variam de $2,98 \times 10^{-9} \mathrm{~m}^{2} . \mathrm{s}^{-1}$ para o combinado $\mathrm{C}_{3}$ na temperatura de secagem de $40{ }^{\circ} \mathrm{C}$ a $8,91 \times 10^{-9} \mathrm{~m}^{2} . \mathrm{s}^{-1}$ para o combinado $\mathrm{C}_{1}$ na temperatura de secagem de $70{ }^{\circ} \mathrm{C}$, e esta relação do coeficiente de difusão com a temperatura de secagem pode ser descrita pela equação de Arrhenius, que apresenta uma energia de ativação variando de 12,65 a 21,64 Kj mol ${ }^{-1}$ para a difusão líquida no processo de secagem entre os combinados de polpa de noni com umbu.

\section{Agradecimentos}

A Coordenação de Aperfeiçoamento de Pessoal de Nível Superior (CAPES) pelo apoio financeiro indispensável na execução do presente trabalho.

\section{Referências}

Alves, J. J. L., \& Rodovalho, R. S. (2016). Cinética de secagem em camada de espuma da polpa de abacate cv. 'quintal' (Persea americana mill). Revista Agrotecnologia, 6(1), 86-99. 10.12971/2179-5959/agrotecnologia.v7n1p86-98

Araújo, W. D., Goneli, A. L. D., Corrêa, P. C., Hartmann Filho, C. P., \& Martins, E. A. S. (2017). Modelagem matemática da secagem dos frutos de amendoim em camada delgada. Ciência Agronômica, 48(3), 448-457. 10.5935/1806-6690.20170052

Baptestini, F. M., Corrêa, P. C., Junqueira, M. S., Ramos, A. M., Vanegas, J. D. B., \& Costa, C. F. (2015). Modelagem matemática da secagem de espuma de graviola. Revista Brasileira de Engenharia Agrícola e Ambiental, 9(12), 1203-1208. http://dx.doi.org/10.1590/1807-1929/agriambi.v19n12p1203-1208

Botelho, F. M., Garcia, T. R. B., Viana, J. L., Botelho, S. C. C., \& Sousa, A. M. B. (2015). Cinética de secagem e determinação do coeficiente de difusão efetivo de grãos de sorgo. Revista Brasileira de Milho e Sorgo, 14(2), 260-271. https://doi.org/10.18512/1980-6477/rbms.v14n2p260-272

Casarin, F., Mendes, C. E., Lopes, T. J., \& Moura, N. F. (2016). Planejamento experimental do processo de secagem da amora-preta (Rubus sp.) Para a produção de farinha enriquecida com compostos bioativos. Brazilian Journal of Food Technology, 19(1), E2016025. https://doi.org/10.1590/1981-6723.2516. 
Castro, D. S., Silva, L. M. M., Moreira, I. S., Sousa, F. C., Silva, W. P., \& Almeida, F. A. C. (2016). Análise e descrição matemática da secagem de polpa de taturubá (Pouteriamacrophylla (lam.) Eyma.) em camada fina. Engevista, 18(2), 309-317. https://doi.org/10.22409/engevista.v18i2.734

Celma, A. R., Cuadros, F., \& López-Rodríguez, F. (2011). Convective drying characteristics of sludge from treatment plants in tomato processing industries. Food and Bioproducts Processing, 90(2), 224-234. 10.1016/j.fbp.2011.04.003

Corrêa, P. C., Oliveira, G. H. H., Botelho, F. M.; Goneli, A. L. D., \& Carvalho, F. M. (2010) Modelagem matemática e determinação das propriedades termodinâmicas do café (Coffea arabica L.) durante o processo de secagem. Revista Ceres, 57(5), 595-601. https://doi.org/10.1590/S0034-737X2010000500005

Dieb, J. T., Gurgel, C. M., Dantas, T. P., \& Medeiros, M. F. D. (2015). Secagem da polpa de graviola pelo processo foam-mat e avaliação sensorial do produto obtido. Revista Tecnologia e Informação, 2(2), 24-31. https://repositorio.unp.br/index.php/tecinfo/article/view/1157

Ferreira, L. F. D., Pirozi, M. R., Ramos, A. M., \& Pereira, J. A. M. (2012). Modelagem matemática da secagem em camada delgada de bagaço de uva fermentado. Pesquisa Agropecuária Brasileira, 47(6), 855-862. http://dx.doi.org/10.1590/S0100-204X2012000600017

Goneli, A. L. D., Vieira, M. C., Vilhasanti, H. C. B., \& Gonçalves, A. A. (2014). Modelagem matemática e difusividade efetiva de folhas de aroeira durante a secagem. Pesquisa Agropecuária Tropical, 44(1), 56-64. https://doi.org/10.1590/S1983-40632014000100005

Gonçalves, J. Q., Silva, M. A. P., Plácido, G. R., Caliari, M., Silva, R. M., Moura, L. C., \& Souza, D. G. (2016). Secagem da casca e polpa da banana verde (Musa acuminata): propriedades físicas e funcionais da farinha. Global Science and Technology, 9(3), 62-72. https://rv.ifgoiano.edu.br/periodicos/index.php/gst/index

Guedes, A. M., \& Faria, L. J. G. (2000). Determinação da constante de secagem de urucum (Bixa orellana L.) em secador convectivo de leito fixo. Revista Brasileira de Produtos Agroindustriais, 2(1), 73-86. http://dx.doi.org/10.15871/1517-8595/rbpa.v2n1p73-86

Gupta, S., Cox, S., \& Abu-ghannam, N. (2011). Effect of different drying temperatures on the moisture and phytochemical constituents of edible irish brown seaweed. LWT-Food Science and Technology, 44(5), 1266-1272. 10.1016/j.1wt.2010.12.022

Moreira, R., Chenlo, F., \& Torres, M. D., Vallejo, N. (2008). Thermodynamic analysis of experimental sorption isotherms of loquat and quince fruits. Journal of Food Engineering, 88(4), 514-521. 10.1016/j.jfoodeng.2008.03.011

Moreira, I. S., Silva, W. P., Castro, D. S., Silva, L. M. M., Gomes, J. P., \& Silva, C. M. D. P. S. (2018). Production of kiwi snack slice with different thickness: drying kinetics, sensory and physicochemical analysis. Australian Journal of Crop Science, 12(5), 778-787. 10.21475/ajcs.18.12.05.PNE925

Ojediran, J. O., \& Raji, A. O. (2010). Thin layer drying of millet and effect of temperature on drying characteristics. International Food Research Journal, 17(1), 1095-1106. Obtido em https://www.researchgate.net/publication/286805099

Oliveira, G. H. H., Aragão, D. M. S., Oliveira, A. P. L. R., Silva, M. G., \& Gusmão, A. C. A. (2015). Modelagem e propriedades termodinâmicas na secagem de morangos. Brazilian Journal of Food Technology, 18(4), 314-321. https://doi.org/10.1590/1981-6723.5315

Pê, P. R., Carneiro, G. G., Pê, F. R.; Castro, D. S., Silva, D. R. S., \& Marques, L. F. (2016). Secagem de polpa de caqui pelo método de camada de espuma. Holos, 4(32), 77-85. 10.15628/holos.2016.2444

Silva Filho, E. D., Alves, G. S., Duarte, M. T. L., \& Madureira, I. A. (2016). Modelagem matemática para descrição da cinética de secagem da polpa da manga cv. Espada. Revista Principia, 1(31), 09-14. http://dx.doi.org/10.18265/1517-03062015v1n31p7-12

Silva, L. M., Sousa, F. C., Castro, D. S., Gomes, J. P., \& Almeida, F. A. C. (2015). Estudo experimental da secagem de polpa de achachairu em camada fina. Gaia Scientia, 9(1), 151-155. https://periodicos.ufpb.br/index.php/gaia/article/view/18136/14849

Silva, R. B., Silva, F. S., Porto, A. G., \& Alves, A. P. (2016). Estudo da cinética de secagem de polpa de carambola. Revista Brasileira de Tecnologia Agroindustrial, 10(2), 2069-2080. 10.3895/rbta.v10n2.3261

Silva, W. P., Silva, C. M. D. P. S., Gama, F. J. A., \& Gomes, J. P. (2014). Mathematical models to describe thin-layer drying and to determine rate of whole bananas. Journal of the Saudi Society of Agricultural Sciences, 13(1), 67-74. https://doi.org/10.1016/j.jssas.2013.01.003

Sousa, K. A., Resende, O., Chaves, T. H., \& Costa, L. M. (2011). The drying kinetics of forage turnips (Raphanus sativus L.). Revista Ciência Agronômica, 42(4), 883-892. https://doi.org/10.1590/S1806-66902011000400009

Sousa, F. C., Silva, L. M. M., Lemos, D. M., Moreira, I. S., Lins, A. D. F., Castro, D. S., \& Rocha, A. P. T. (2015). Secagem de resíduos de Spondias sp. em camada fina. Revista agropecuária técnica, 36(1), 197-202. https://doi.org/10.25066/agrotec.v36i1.24449

Sousa, F. C., Sousa, E. P., Silva, L. M. M., Martins, J. J. A., Gomes, J. P., \& Rocha, A. P. T. (2011). Modelagem matemática para descrição da cinética de secagem de polpa de oiti. Revista Educação Agrícola Superior, 26(2), 108-112. http://www.abeas.com.br/wt/revista.php

Zogzas, N. P., Maroulis, Z. B., \& Marinoskouris, D. (1996). Moisture diffusivity data compilation in foodstuffs. Drying Technology, 14(10), 2225-2253. https://doi.org/10.1080/07373939608917205 